Reihe Geschichtswissenschaft Band 44 


\section{Arbeitslosigkeit im NS-Staat}

Das Beispiel München

\section{Claudia Brunner}

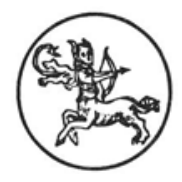

Centaurus Verlag \& Media UG 1997 
Die Autorin, Claudia Brunner, arbeitet als Politikwissenschaftlerin und Historikerin in München. Veröffentlichungen:

Arbeitslosigkeit in München 1927 bis 1933. Kommunalpolitik in der Krise, München 1992; »Bettler, Schwindler, Psychopathen«: Die »Asozialen«-Politik des Münchner Wohlfahrtsamtes in den frühen Jahren der NS-Zeit (1933 bis 1936), München 1993; Frauenarbeit im Männerstaat. Wohlfahrtspflegerinnen im Spannungsfeld kommunaler Sozialpolitik in München 1919-1938, Pfaffenweiler 1994.

Dieses Buch wurde gedruckt mit freundlicher Unterstützung der Deutschen Forschungsgemeinschaft.

Die Deutsche Bibliothek - CIP-Einheitsaufnahme

Brunner, Claudia :

Arbeitslosigkeit im NS-Staat : das Beispiel München /

Claudia Brunner. - Pfaffenweiler : Centaurus Verl.-Ges., 1997

(Reihe Geschichtswissenschaft ; Bd. 44)

ISBN 978-3-8255-0128-0 ISBN 978-3-86226-289-2 (eBook)

DOI $10.1007 / 978-3-86226-289-2$

NE: GT

\section{ISSN 0177-2767}

Alle Rechte, insbesondere das Recht der Vervielfältigung und Verbreitung sowie der Übersetzung, vorbehalten. Kein Teil des Werkes darf in irgendeiner Form (durch Fotokopie, Mikrofilm oder ein anderes Verfahren) ohne schriftliche Genehmigung des Verlages reproduziert oder unter Verwendung elektronischer Systeme verarbeitet, vervielfältigt oder verbreitet werden.

C CENTAURUS-Verlagsgesellschaft mit beschränkter Haftung, Pfaffenweiler 1997

Umschlagabbildung: Reichsautobahnbau München-Salzburg, 1934. Arbeiter bei der Essensausgabe. Foto: Stadtarchiv München

Satz: Martin Turczak, München 
Martin T. gewidmet 


\section{Inhaltsverzeichnis}

Seite

Abkürzungsverzeichnis $\quad \mathrm{X}$

Einleitung 1

1. Zur Ideologie des nationalsozialistischen Arbeitsstaats:

die Durchsetzung des Arbeits- und Leistungszwangs

2. Die Bedeutung von Propagandakampagnen im Rahmen der „Arbeitsschlacht“

2.1 Schwarzarbeit

2.2 Hand- und Maschinenarbeit 26

2.3 ,Doppelverdiener“ $\quad 29$

3. Die Entwicklung des „Gesetzes über Arbeitsvermittlung und Arbeitslosenversicherung" (AVAVG) und der Arbeitslosenunterstützung

4. Die Situation bei der lokalen Arbeitslosigkeit anhand ausgewählter Gruppen: Arbeiter und Angestellte, ,alte Kämpfer“, Frauen, Jugendliche

5. Die Entwicklung der staatlichen Arbeitsverwaltung

5.1 Arbeitsamt München

5.2 Landesarbeitsamt Bayern

6. Der staatliche Zugriff auf Arbeitslose und Arbeitnehmer

6.1 Erwerbslosenfortbildung, Erwerbslosenbetreuung

6.2 Deutsche Arbeitsfront (DAF)

6.3 Arbeitsbuch

6.4 Arbeitskräftemangel, Arbeitseinsatz 
7. Vom freiwilligen Arbeitsdienst zur Arbeitsdienstpflicht

7.1 „Münchener Jugenddienst“

7.2 Freiwilliger Arbeitsdienst (FAD) 103

7.3 Reichsarbeitsdienst (RAD), „Arbeitsdank“ 111

7.4 Reichsarbeitsdienst für die weibliche Jugend (RADwJ) 120

8. Staatliche Sofortmaßnahmen zur Verringerung der

Massenarbeitslosigkeit

8.1 Arbeitsbeschaffungsprogramme

8.2 Notstandsarbeiten 144

8.3 Reichsautobahnbau 152

8.4 Landhilfe, Landjahr, HJ-Landdienst 167

9. Kommunale Sozialpolitik unter totalitären Vorzeichen: die Eliminierung von Wohlfahrtserwerbslosen und Fürsorgeempfängern

10. Die Etablierung der kommunalen Arbeitsfürsorge

10.1 Pflichtarbeit für Männer

10.2 Pflichtarbeit für Frauen

220

10.3 Fürsorgearbeit

11. Die Verschärfung der „Asozialen“-Politik

11.1 „Arbeitsscheu“

11.2 Arbeitsanstalten

12. Individuelle und kollektive Strategien zur Konfliktverarbeitung und Anpassung

12.1 Selbstmord, Selbstmordversuch, Ehe- und Geburtenentwicklung 277

12.2 Widerstand, Sabotage, Denunziation

13. Die Ernährungs- und Versorgungslage der Münchner Bevölkerung

13.1 Städtische Suppenanstalten

13.2 Nahrungs- und Konsumgüter 
14. Flankierende sozial- und wirtschaftspolitische Maßnahmen von Staat, Kommune und Partei

14.1 „Notwerk der deutschen Jugend“

14.2 Ehestandsdarlehen, Kinderbeihilfen, Kinder- und Mütterverschickung, NS-Hilfswerk „Mutter und Kind“, Reichsmütterdienst

14.3 Der Abbau der Selbsthilfe

14.4 Vier-Jahres-Plan, Preisüberwachung

15. Das Sammel- und Spendenwesen als gesellschaftlicher und politischer Integrationsfaktor

15.1 Winterhilfswerk des Deutschen Volkes (WHW)

15.2 Münchner Nothilfe Lotterie, Hitler-Freiplatz-Spende, NSDAP-Arbeitsbeschaffungslotterie, NSV

Schlußbetrachtung

Literatur

Tabellenverzeichnis 


\section{Abkürzungsverzeichnis}

a.a.O. am angegebenen Ort

Abb. Abbildung

Abt. Abteilung

ADGB Allgemeiner Deutscher Gewerkschaftsbund

AG Amtsgericht

A.G. Aktiengesellschaft

Alu. /ALU Arbeitslosenunterstützung

AVAVG Gesetz über Arbeitsvermittlung und Arbeitslosenversicherung

BA Bundesarchiv Koblenz

Bd./Bde. Band/Bände

Bearb. Bearbeitung

betr. betreffend

BFV Bezirksfürsorgeverband

Bl. Blatt

BMW Bayerische Motoren Werke

DAF Deutsche Arbeitsfront

dgl. dergleichen

d.V. die Verfasserin

Fa. Firma

Ffm. $\quad$ Frankfurt am Main

g Gramm

Gestapo Geheime Staatspolizei

gez. gezeichnet

$\mathrm{GmbH} \quad$ Gesellschaft mit beschränkter Haftung

HJ Hitler-Jugend

Hg./hg. Herausgeber/herausgegeben

IHK Industrie- und Handelskammer

Jg. Jahrgang

km Kilometer

Koord. Koordination

KPD Kommunistische Partei Deutschlands

Kru. /KRU Krisenunterstützung

$\mathrm{KZ} / \mathrm{KL} \quad$ Konzentrationslager

LRA Landratsamt 
M

MInn

Mio.

Mrd.

MWi

$\mathrm{Nr}$.

NS

NSBO

NSDAP

NSKK

NSLB

NSV

Obb.

o.D.

o.J.

o.O.

$\mathrm{Pg}$.

Pol.Dir.

qm

RAB

RAD

RADwJ

RAM

RFSS

RFV

$\mathrm{RM}$

Rpf.

RSHA

S.

SA

SPD

SS

StK

tw.

u.

u.k.

unpag.

WBA.
Mark

Innenministerium

Million

Milliarde

Wirtschaftsministerium

Nummer

Nationalsozialismus

Nationalsozialistische Betriebszellen-Organisation

Nationalsozialistische Deutsche Arbeiterpartei

Nationalsozialistisches Deutsches Kraftfahrer-Korps

Nationalsozialistischer Lehrerbund

Nationalsozialistische Volkswohlfahrt

Oberbayern

ohne Datum

ohne Jahr

ohne Ort

Parteigenosse

Polizeidirektion München

Quadratmeter

Reichsautobahn

Reichsarbeitsdienst

Reichsarbeitsdienst für die weibliche Jugend

Reichsarbeitsministerium

Reichsführer-SS

Reichsverordnung über die Fürsorgepflicht

Reichsmark

Reichspfennig

Reichssicherheitshauptamt

Seite

Sturmabteilung

Sozialdemokratische Partei Deutschlands

Schutzstaffel

Staatskanzlei

teilweise

und

unabkömmlich

unpaginiert

Wohlfahrtsbezirksamt 
WE. Wohlfahrtserwerbslose

Wolu. Wohlfahrtsunterstützung

WHW Winterhilfswerk

zit. zitiert 